\title{
Fiskalische Effekte der Cannabis-Legalisierung
}

Die neue Bundesregierung hat angekündigt, den Konsum von Cannabis in Deutschland zu legalisieren. Wörtlich heißt es im Koalitionsvertrag: „Wir führen die kontrollierte Abgabe von Cannabis an Erwachsene zu Genusszwecken in lizenzierten Geschäften ein. Dadurch wird die Qualität kontrolliert, die Weitergabe verunreinigter Substanzen verhindert und der Jugendschutz gewährleistet. Das Gesetz evaluieren wir nach vier Jahren auf gesellschaftliche Auswirkungen." (SPD, Bündnis 90/Die Grünen und FDP, 2021).

Unter Ökonomen ist die Forderung nach einer Legalisierung von Cannabis nichts Neues. Milton Friedman (1972) hatte sie schon 1972 gefordert. Zahlreiche andere Ökonomen haben diese Forderung in der Folgezeit immer wieder aufgegriffen, darunter auch zahlreiche Nobelpreisträger wie George Akerlof und Vernon Smith 2005 (Debusmann, 2008) oder Kenneth Arrow, Christopher Pissarides und Thomas Schelling 2014 (Schomberg, 2014). Die Vorteile einer Legalisierung bestehen dabei vor allem darin, dass so faktisch ein besserer Jugend- und Gesundheitsschutz zu gewährleisten ist als durch Prohibition. Auch findet sich bisher keine belastbare Evidenz dafür, dass der Cannabiskonsum aufgrund von Legalisierungsmaßnahmen dauerhaft und systematisch ansteigt, wie der Wissenschaftliche Dienst des Deutschen Bundestages (2019) in einer Zusammenfassung der vorliegenden Studienlage ausgeführt hat.

Nachdem bis 2017 weder Informationen über die Kosten vorlagen, die durch die Verfolgung von Cannabisdelikten entstanden sind, noch über mögliche Steuereinnahmen im Falle einer Legalisierung (Deutscher Bundestag, 2017), haben Haucap et al. (2018; 2019) dazu erstmalig Zahlen vorgelegt. Diese Analyse ist nun aktualisiert worden (Haucap und Knoke, 2021a) und wird im Folgenden zusammen-

(C) Der/die Autor:in 2021. Open Access: Dieser Artikel wird unter der Creative Commons Namensnennung 4.0 International Lizenz veröffentlicht (creativecommons.org/licenses/by/4.0/deed.de).

Open Access wird durch die ZBW - Leibniz-Informationszentrum Wirtschaft gefördert.

Prof. Dr. Justus Haucap ist Direktor des Düsseldorf Institute for Competition Economics (DICE) an der Heinrich-Heine-Universität in Düsseldorf.

Leon Knoke ist dort Research Assistant. gefasst. Argumente für und wider eine Legalisierung sind nicht Gegenstand dieses Beitrags, sie werden von Haucap und Knoke (2021b) gesondert erörtert.

Zur Ermittlung der möglichen Staatseinnahmen muss der zu erwartende Gesamtbedarf an Cannabis auf einem legalisierten Markt eingeschätzt werden. Naturgemäß liegen keine amtlichen Zahlen über die heute konsumierte Cannabismenge vor. Anhand sogenannter Prävalenzdaten lassen sich jedoch Rückschlüsse ziehen, wie viel Cannabis in Deutschland konsumiert wird. Diese Prävalenzdaten basieren auf den sogenannten Epidemiologischen Suchtsurveys, die in Deutschland bisher im Dreijahres-Rhythmus durchgeführt werden (IFT, 1995-2018). Erhoben wird dazu bei einer repräsentativen Stichprobe, welcher Anteil der Bevölkerung in Deutschland innerhalb einer bestimmten Altersgruppe eine bestimmte Substanz mindestens einmal in den letzten 30 Tagen (30-Tages-Prävalenz), mindestens einmal in den letzten zwölf Monaten (12-Monats-Prävalenz) oder mindestens einmal im ganzen Leben (Lebenszeit-Prävalenz) konsumiert haben. Befragt werden die Teilnehmer seit 1980 nach ihrem Konsum von Alkohol, Tabak, illegalen Drogen sowie Medikamenten.

Mit einer bisherigen Lebenszeit-Prävalenz von 31,9\% und einer 12-Monats-Prävalenz von 8,3\% lag Cannabis 2018 deutlich vor dem Konsum von Drogen wie beispielsweise Kokain, Amphetaminen oder Ecstasy, deren Lebenszeit-Prävalenzen allesamt unter $5 \%$ liegen (Seitz et al., 2019). Abbildung 1 veranschaulicht die Entwicklung des Cannabis-Konsums von 1995 bis 2018 anhand der Lebenszeit-, 12-Monats- und der 30-Tages-Prävalenz in Deutschland. Für die Analysen in der weiteren Studie wurden ebenfalls die Daten der Altersgruppe von 60 bis 64 berücksichtigt, die in den epidemiologischen Suchtsurveys inzwischen auch erhoben werden.

Auf Basis der Prävalenzdaten des Epidemiologischen Suchtsurveys 2018 und Daten des Statistischen Bundesamtes zur Bevölkerung nach Alters- und Geburtsjahren wurden für die 30-Tages-Prävalenz für 2018 etwa 1,5 Mio. Personen zwischen 18 und 64 Jahren berechnet, die in den letzten 30 Tagen Cannabis konsumiert haben (Haucap und Knoke, 2021a, 17 und Anhang 6.1). Für die 12-Monats-Prävalenz wurden für 2018 eine Zahl von gut 3,6 Mio. Personen zwischen 18 und 64 Jahren ermittelt, die in den zwölf Monaten vor der Befragung Cannabis konsumiert haben. Diese Zahl spiegelt die Gesamtzahl an Personen wider, die heute in Deutschland gelegentlich bis regelmäßig Cannabis konsumieren und zur Ermittlung des jährlichen Gesamtbedarfs an Cannabis zugrunde zu legen ist. 


\section{Abbildung 1}

Entwicklung der Prävalenz von Cannabis in Deutschland für 18- bis 59-Jährige

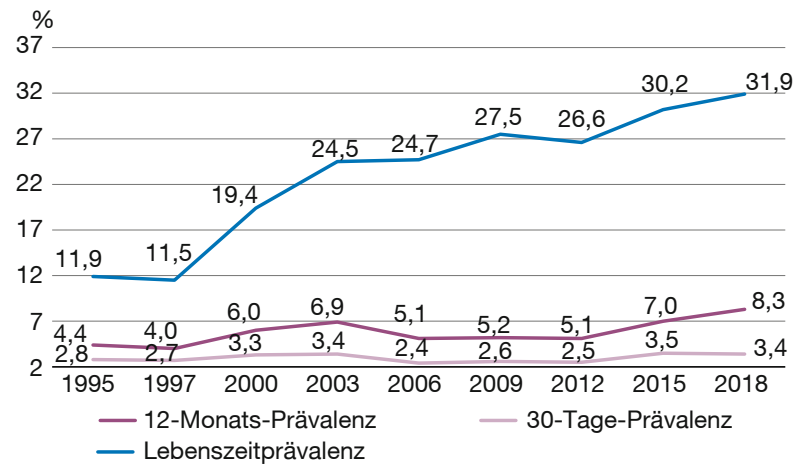

Quelle: Seitz et al. (2019), Tabellen 2, 4 und 6.

Es wurden alle Konsumierende, die mindestens einmal im vergangenen Jahr konsumiert haben, in drei Nutzergruppen eingeteilt: 1. Gelegenheitskonsumierende, 2. regelmäBig Konsumierende und 3. Intensivnutzer. Gemäß Light et al. (2014) nutzen Gelegenheitskonsumierende Cannabis weniger als einmal pro Monat. Regelmäßig Konsumierende konsumieren Cannabis zwischen ein und 20 Mal pro Monat und Intensivnutzer konsumieren an mehr als 20 Tagen pro Monat bis täglich. Die Aufteilung der Konsumierenden in die jeweiligen Nutzergruppen wurde auf Basis von Daten zu Konsumfrequenzen aus dem Epidemiologischen Suchtsurvey 2018 vorgenommen (Haucap und Knoke, 2021a, 17-18 und Anhang 6.2). Auf Basis der erhobenen Daten zu Konsumfrequenzen ergibt sich, dass rund $63 \%$ aller Konsumierenden in Deutschland Gelegenheitskonsumierende sind, $25 \%$ regelmäßige Konsumierende und rund $12 \%$ Intensivnutzer. Um den Gesamtbedarf bestimmen zu können, wurden die rund 3,6 Mio. Konsumierenden auf die drei zuvor definierten Konsumgruppen (gelegentlich, regelmäßig, intensiv) aufgeteilt. Für alle drei Konsumgruppen wurden dann in einem nächsten Schritt aus der Literatur Annahmen über Konsumtage pro Jahr und Konsummengen pro Tag abgeleitet, auf deren Basis der Gesamtbedarf an Cannabis ermittelt wird (Haucap und Knoke, 2021a, 18-19). Hierbei wurden drei Szenarien gebildet, um verschiedene Konsummuster der einzelnen Konsumgruppen abzubilden. In einem sehr konservativen Ansatz werden die Konsumtage pro Jahr und Konsummengen pro Tag sehr vorsichtig angesetzt, im nicht konservativen Ansatz werden großzügigere Annahmen getroffen. Im konservativen Szenario wird die Mitte beider Ansätze zugrunde gelegt. Für die Konsummengen pro Tag werden Schätzungen aus den USA zugrunde gelegt (Kilmer et al., 2013; Light et al., 2014), auf die sich im Übrigen auch der französische Sachverständigenrat für Wirtschaftsfragen in seiner Schätzung des potenziellen Steueraufkommens für Frankreich stützt (Conseil d'analyse économique, 2019).
Der ermittelte Gesamtbedarf muss sodann um Effekte wie Bevölkerungswachstum, bisheriges Under-Reporting, illegaler Konsum Minderjähriger ${ }^{1}$ und touristische Nachfrage angepasst werden (Haucap und Knoke, 2021a, 19-25 und Anhang 6.3). Für Deutschland wurde so eine Gesamtzahl von fast 5 Mio. Konsumierenden abgeleitet und ein jährlicher Gesamtbedarf von etwa 377 t Cannabis.

In einem zweiten Ansatz wurde der Gesamtbedarf aus einem an deutsche Prävalenzdaten angepassten Pro-Kopf-Konsum in Frankreich bestimmt. So wurden die unterstellten Annahmen bezüglich der Konsummuster unterschiedlicher Konsumgruppen im ersten Ansatz überprüft, um damit ein möglichst aussagekräftiges Ergebnis im Hinblick auf einen tatsächlich anzunehmenden Gesamtbedarf in Deutschland zu ermitteln. Die Ableitung des Gesamtbedarfs in Deutschland über den Pro-Kopf-Konsum in Frankreich ist somit als Robustheitscheck für den ermittelten Gesamtbedarf in Deutschland über deutsche Prävalenzdaten anzusehen. Basierend auf dem jährlichen Pro-Kopf-Konsum für die französischen Nutzergruppen folgt ein jährlicher Gesamtbedarf von gut $393 \mathrm{t}$ in Deutschland (Haucap und Knoke, 2021a, 23-25 auf Basis der Annahmen von Conseil d'analyse économique, 2019).

In einem dritten Ansatz haben wir den Gesamtbedarf über einen für Deutschland angepassten Pro-Kopf-Konsum der aus Colorado vorliegenden Daten vorgenommen und so einen Bedarf von gut $545 \mathrm{t} / \mathrm{Jahr}$ ermittelt (Haucap und Knoke, 2021a, 25-33). Colorado eignet sich gut für einen Vergleich, da es seit der Legalisierung in Colorado 2014 amtliche Statistiken über das tatsächliche Konsumverhalten sowie jährliche Verkaufsmengen gibt. Um jedoch eine eher konservative Schätzung zu erhalten, sind wir für die weiteren Berechnungen von einem Gesamtbedarf für Deutschland von „nur“ $400 \mathrm{t}$ ausgegangen.

Auf dieser Basis und ausgehend von einem durchschnittlichen Bruttopreis von 10 Euro/g wurden insgesamt Steuereinnahmen für einzelne Steueraufkommensarten in Höhe von 2,8 Mrd. Euro ermittelt (Haucap und Knoke, 2021a, 33-45). Der Preis von 10 Euro ergibt sich daraus, dass dies in etwa dem heutigen Schwarzmarktpreis entspricht (www.graspreis.de) und der Preis auf dem legalen Markt nicht zu sehr über dem Schwarzmarktpreis liegen darf, um den Schwarzmarkt effektiv einzudämmen. Zudem wurden für Deutschland vergleichbare Produktions- und Marktstrukturen wie in Colorado angenommen, um Annahmen über Produktionskosten, entstehende Gewerbezahlen sowie vollzeitäquivalente Arbeitsplätze treffen zu können. In Colorado kommen beispielsweise etwa 69 vollzeitäquiva-

1 Wir nehmen an, dass es weiterhin einen illegalen Konsum durch Minderjährige geben wird, jedoch etwa die Hälfte davon sich Cannabis illegal im legalisierten Markt besorgt und die Ware somit versteuert wird. 
lente Arbeitsplätze auf jede verkaufte Tonne Cannabis. Für Deutschland mit einem geschätzten Gesamtbedarf von $400 \mathrm{t}$ würden sich entsprechend rund 27.600 vollzeitäquivalente Arbeitsplätze ergeben. Auf dieser Basis wäre eine Cannabis-Steuer von 4,50 Euro/g möglich, ohne den Zielpreis von 10 Euro/g inklusive aller Steuern nennenswert zu überschreiten. Als mögliches Steueraufkommen ergibt sich dann das Aufkommen der Cannabis-Steuer (1,8 Mrd. Euro), der $19 \%$ igen Umsatzsteuer (650 Mio. Euro), Gewerbe- und Körperschaftsteuer (85 Mio. Euro) sowie Lohnsteuer (280 Mio. Euro) auf. Zusätzlich wurde das Sozialversicherungsaufkommen (526 Mio. Euro) geschätzt.

Im Rahmen der Rechtsdurchsetzung belaufen sich mögliche Kosteneinsparungen auf insgesamt rund 1,36 Mrd. Euro (Haucap und Knoke, 2021a, 46-55), die sich wie folgt aufteilen: Bei der Polizei lassen sich durch eine Legalisierung mögliche Kosteneinsparungen von 1,1 Mrd. Euro ermitteln. Die Kosten der Staatsanwaltschaft, der Gerichte und des Justizvollzugs werden auf rund 313 Mio. Euro geschätzt. Es ist zu berücksichtigen, dass es durch die Cannabis-Legalisierung nicht unbedingt zu einem Stellenabbau kommen wird. Vielmehr ist davon auszugehen, dass freigewordene Kapazitäten für andere Fälle genutzt werden können. Sofern diese Aktivitäten für die Steuerzahler mindestens einen Wert haben wie die damit verbundenen Kosten, die dann nicht mehr zur Verfolgung von Cannabis-Delikten aufgewendet werden müssen, entsteht hier ein volkswirtschaftlicher Mehrwert.

Nicht einbezogen in unsere Berechnung haben wir zum einen die Kosten des Lizenzsystems in einem legalisierten Markt. Wir unterstellen hier, dass sich diese Kontrollkosten durch die Einnahmen aus den fälligen Lizenzgebühren, die wir ebenfalls unberücksichtigt lassen, selbst tragen sollten. Zum anderen haben wir nicht ermitteln können, wie sich die Gesundheitskosten nach einer Legalisierung entwickeln. Gehen wir aber davon aus, dass der problematische Konsum, insbesondere bei Teenagern, sogar eher sinken als zunehmen wird - so wie dies in einigen US-Bundesstaaten heute zu beobachten ist (Coley et al., 2021) - so entstehen auch keine zusätzlichen Gesundheitskosten. Im Gegenteil ist dann sogar eher von sinkenden Gesundheitskosten auszugehen, weil gesundheitliche Schäden, die heute durch Streckmittel und Verunreinigungen entstehen, in einem legalisierten Markt weitgehend entfallen dürften. Insgesamt ergibt sich ein Betrag von rund 4,7 Mrd. Euro, der durch eine Legalisierung von Cannabis realisiert werden kann, einerseits durch zusätzliche Einnahmen (Steuern und Sozialabgaben) und andererseits durch eingesparte Ausgaben (vgl. Tabelle 1). Aus ökonomischer Sicht sollten die fiskalischen Auswirkungen der Legalisierung aber nicht das Hauptargument für die Legalisierung sein, sondern eher eine angenehme Nebenwir-
Tabelle 1

Überblick über Steuereinnahmen pro Steuerart in Euro

Steuerart/

Einsparungen Rechtsdurchsetzung

Einnahmen/Einsparungen

\begin{tabular}{lc}
\hline Cannabis-Steuer & 1.800 .000 .000 \\
\hline Umsatzsteuer & 650.000 .000 \\
\hline Gewerbesteuer & 25.978 .909 \\
\hline Körperschaftssteuer & 58.867 .248 \\
\hline Lohnsteuer & 279.892 .152 \\
\hline Sozialversicherungsaufkommen & 525.949 .740 \\
\hline Eingesparte Polizeikosten & 1.051 .185 .370 \\
\hline Eingesparte Gerichtskosten & 272.302 .843 \\
\hline Eingesparte Justizvollzugskosten & 41.111 .200 \\
\hline Gesamt & 4.705 .287 .462 \\
\hline
\end{tabular}

Quelle: eigene Berechnungen.

kung. Wichtigstes Argument ist vielmehr der verbesserte Jugend- und Gesundheitsschutz in einem legalen Markt. Eine Marktordnung, die wirksamen Jugend- und Verbraucherschutz gewährleistet und den Schwarzmarkt schnell austrocknet, muss daher sorgfältig gestaltet werden.

\section{Literatur}

Coley, L. R., C. Kruzik, M. Ghiani, N. Carey, S. S. Hawkins und C. F. Baum (2021), Recreational Marijuana Legalization and Adolescent Use of Marijuana, Tobacco, and Alcohol, Journal of Adolescent Health, 69, 41-49.

Conseil d'analyse économique (2019), Une filière du cannabis en France, Focus, 034-2019.

Debusmann, B. (2008), REUTERS COLUMN-Einstein, insanity and the war on drugs, Reuters, 3. Dezember.

Deutscher Bundestag (2017), Drucksache 19/310 vom 21. Dezember.

Friedman, M. (1972), Prohibition and Drugs, Newsweek, 1. Mai, 104.

Haucap, J. (2019), Auch der Fiskus profitiert: Der Nutzen einer Cannabislegalisierung in Deutschland, Alternativer Sucht- und Drogenbericht, 6, 112-119.

Haucap, J., C. Kehder, M. Feist und J. Slowik (2018), Die Kosten der Cannabis-Prohibition in Deutschland.

Haucap, J. und L. Knoke (2021a), Fiskalische Auswirkungen einer Cannabislegalisierung in Deutschland: Ein Update.

Haucap, J. und L. Knoke (2021b), Warum und wie die Liberalisierung des Cannabis-Marktes in Deutschland erfolgen sollte, im Erscheinen.

IFT - Institut für Therapieforschung (1995-2018), Epidemiologische Suchtsurveys.

Kilmer, B., J. P. Caulkins, G. Midgette, L. Dahlkemper, R. J. MacCoun und R. L. Pacula (2013), Before the Grand Opening: Measuring Washington State's Marijuana Market in the Last Year Before Legalized Commercial Sale, RAND Drug Policy Research Center.

Light, M. K., A. Orens, B. Lewandowski und T. Pickton (2014), Market Size and Demand for Marijuana in Colorado, Papier für das Colorado Department of Revenue, 17.

Schomberg, W. (2014), Nobel economists, others urge end to ,war on drugs', Reuters, 6. Mai.

Seitz, N.-N., L. Böttcher, J. Atzendorf, C. Rauschert und L. Kraus (2019), Kurzbericht Epidemiologischer Suchtsurvey 2018. Tabellenband: Trends der Prävalenz des Konsums illegaler Drogen und Drogenmissbrauch und -abhängigkeit nach Geschlecht und Alter 1990-2018.

SPD, Bündnis 90/Die Grünen und FDP (2021), Koalitionsvertrag 20212025, Mehr Fortschritt wagen, Zeilen 2890-2893.

Wissenschaftliche Dienste des Deutschen Bundestages (2019), Sachstand: Legalisierung von Cannabis Auswirkungen auf die Zahl der Konsumenten in ausgewählten Ländern. 